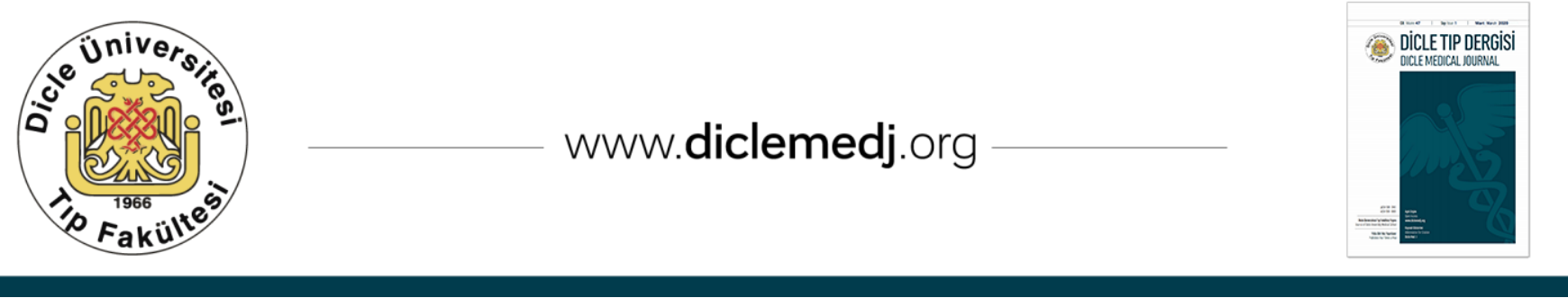

Original Article / Özgün Araştırma

\title{
Bibliometric Analysis of Top 100 Most Cited Articles Related To "Liver Transplantation" in Anesthesia Literature
}

\author{
Selcuk Kayir1 ${ }^{\text {iD }}$, Guvenc Dogan1 ${ }^{\text {iD }}$, Ozgur Yagan1 ${ }^{\text {iD }}$, Volkan Hanci² ${ }^{\text {iD }}$ \\ 1 Department of Anesthesiology and Reanimation, Hitit University Faculty of Medicine, Corum, Turkey \\ 2 Department of Anesthesiology and Reanimation, Dokuz Eylul University School of Medicine, Izmir, Turkey
}

Received: 25.07.2019; Revised: 23.12.2019; Accepted: 07.01.2020

\begin{abstract}
Objective: For treatment of acute liver failure, end-stage liver diseases and liver-sourced malignant tumors, liver transplantation is a treatment method applied as a last resource. In our study, the aim was to assess 100 articles with most citations about liver transplantation in the anesthesia literature.
\end{abstract}

Methods: Our study used the "advanced mode" feature of the "Institute for Scientific Information (ISI) Web of Science (WOS)" search engine. The search was performed on 11.12.2018 and 100 articles with most citations related to liver transplantation were determined. For each article, total citation number, annual citation number, authors, and catalogue information for the study and journal were determined using Web of Science (WOS) and PubMed.

Results: The total mean number of citations for 103 studies with most citations was determined as $75.87 \pm 68.48$. The annual citation numbers for the studies varied from 34.18 to 1.15 , with mean of $4.98 \pm 4.60$. When the areas of focus of the studies are investigated, the first three areas were transfusion practice in 17 articles (16.5\%), thromboelastography in 14 (13.6\%) and hemodynamic monitoring in 10 (9.7\%). The mean number of citations of authors from the European continent was significantly higher than authors from other continents $(\mathrm{p}<0.01)$.

Conclusion: Our study is the first to evaluate and analyze the top 103 articles receiving most citations related to liver transplantation in the anesthesia literature. Bibliographic studies created using scientific search engines are illustrative of the topics of focus for authors and researchers.

Keywords: liver transplantation; citations; bibliometric analysis; web of science

DOI: 10.5798/dicletip.705931

Correspondence / Yazışma Adresi: Selcuk Kayir, Department of Anesthesiology and Reanimation, Hitit University Erol Olcok Training and Research Hospital, Corum, Turkeye-mail:drskayir@gmail.com 


\section{Anestezi Literatüründe "Karaciğer Transplantasyonu” İle İlgili En Çok Atıf Alan 100 Makalenin Değerlendirilmesi}

$\ddot{0} \mathbf{z}$

Amaç: Akut karaciğer yetmezliği, son dönem karaciğer hastalıkları ve karaciğer kaynaklı malign tümörlerin tedavisinde karaciğer transplantasyonu son çare olarak uygulanan tedavi yöntemidir. Çalışmamızda anestezi literatüründe karaciğer transplantasyonu ile ilgili olan ve en çok atıf alan 100 yayının değerlendirilmesi amaçlanmıştır.

Yöntemler: Çalışmamız "Institute for Scientific Information (ISI) Web of Science (WOS)"arama motorunun "advanced mode" özelliği kullanılarak yapıldı. Arama 11.12.2018 tarihinde yapıldı ve arama sonuçlarından karaciğer transplantasyonu ile ilgili en çok atıf alan 100 yayın belirlendi. Her yayının toplam atıf sayısı, yıllık atıf sayısı, çalıșmacıları, çalışmanın ve derginin katalog bilgileri Web of Science (WOS) ve PubMed kullanılarak belirlendi.

Bulgular: En fazla atıf alan 103 çalışmanın toplam atıf sayısının ortalaması 75,87£68,48 olarak belirlendi. Çalışmaların

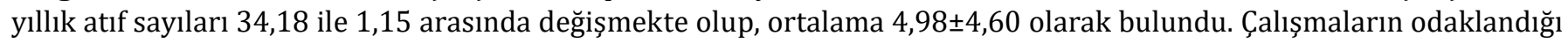
alanlar incelendiğinde ilk üç alanı sırasıyla; transfüzyon pratiği 17 (\%16,5), tromboelastografi $14(\% 13,6)$ ve hemodinamik monitörizasyon $10(\% 9,7)$ gelmekteydi. Avrupa kıtasındaki yazarların atıf sayısı ortalaması Avrupa kıtası dışındaki yazarlardan anlamlı olarak yüksekti $(\mathrm{p}<0.01)$. Derginin kıtası ve derginin indeksi ile toplam ve yıllık atıf sayısı arasında anlamlı bir ilişki belirlenemedi.

Sonuç: Çalışmamız anestezi literatürde karaciğer transplantasyonu ile ilgili en çok atıf alan ilk 103 çalışmanın değerlendirilip analiz edildiği ilk çalışmadır. Bilimsel arama motorlarını kullanılarak oluşturulan bibliografik yazılar, araştırmacılara hangi konulara ağırlık verilmesi konusunda yol göstericidir.

Anahtar kelimeler: karaciğer transplantasyonu; atıf; bibliometrik analiz, web of science.

\section{INTRODUCTION}

The importance of organ transplantation in modern treatment is increasing. Liver transplantation is a treatment method used as a last resource for acute liver failure, end-stage liver diseases and malignant tumors sourced in the liver ${ }^{1}$. Liver transplants from living donors were performed for the first time in Brazil in 1988, but was unsuccessful ${ }^{2}$. After this failure, studies continued rapidly and the first successful liver transplantation was completed in 1990 by Strong et al. in Australia ${ }^{3}$.

Bibliometry is a serial method used to evaluate scientific publications about a certain topic. Bibliometric studies are beneficial to assess the social and scientific importance of a certain scientific field during a certain period 4 . When a scientific article references another scientific article, this is defined as citing that article. Larger numbers of citations received by a scientific article show the power of effect of that article $^{5}$.

According to our research, there is no study investigating citation numbers for international articles about liver transplantation in the anesthesia literature from recent years. This study aims to examine the most cited international articles related to liver transplantation in the anesthesia literature using the Institute for Scientific Information's (ISI) Web of Science (WOS) search engine 6 and to investigate these articles in terms of journal of publication, organizations, countries and authors.

\section{METHODS}

This study used the "advanced mode" feature of the "Institute for Scientific Information (ISI) Web of Science (WOS)" search engine. The search words "SU=anesth* AND TI=transplant* OR SU=anesth* AND TS=transplant* OR 
$\begin{array}{llll}\text { WC }=\text { anesth* }^{*} & \text { AND } & \text { TI }=\text { transplant* } & \text { OR } \\ \text { WC }=\text { anesth* }^{*} & \text { AND } & \text { TS }=\text { transplant* } & \text { OR }\end{array}$ SU=transplant* AND TI=anesth* OR SU=transplant* AND TS=anesth* OR WC= transplant* AND TI=anesth* OR $\mathrm{WC}=$ transplant* AND TS=anesth*" were used. The search was performed on 11.12.2018 and 100 articles with most citations related to liver transplantation were determined. For each article, total citation number, annual citation numbers, authors, and catalogue information for the study and journal were determined using Web of Science (WOS) and PubMed.

Our study included articles from 1975 to December 2018. One-hundred and three publications with most citations were determined from international articles published related to the topic. Since the last four articles had the same citations, a total of 103 articles were analyzed. The list was screened one-by-one to check whether the first authors were listed in any other articles. For each article, total citation number, annual citation numbers, authors, and catalogue information for the study and journal were determined using Web of Science (WOS) and PubMed.

Hitit University Faculty of Medicine Clinical Research Ethics by the presidency of the board

due to the design of the study board approval was not required.

\section{Statistical Analysis}

Data obtained in the research were entered into the SPSS (Statistical Package For Social Sciences, Chicago, IL, USA) 15.0 program for statistical analysis. The Kruskal Wallis test and Mann Whitney U test were used to compare groups. A $\mathrm{p}$ value of less than 0.05 was accepted as significantly different.

\section{RESULTS}

Literature analysis from 1975 to December 2018 with the Web of Science using the designated key words determined a total of 6358 articles. The citation numbers and means for 103 articles with most citations in the field of liver transplantation in the anesthesia literature are given in Table 1. The study with most citations was cited 456 times, while the lowest citation number was 36 . The mean total citation number for the 103 articles with most citations was determined as $75.87 \pm 68.48$. The annual citation numbers for the studies varied from 34.18 to 1.15 , with mean of $4.98 \pm 4.60$.

The distribution of first author names in the studies is given in Table 2. The article with most citations was by Kang Yg, Martın Dj, Marquez J, et al. published in "Anesthesia and Analgesia" journal in 1985 with the title "Intraoperative Changes in Blood-Coagulation and Thrombelastographic Monitoring in Liver Transplantation".

The distribution of first author affiliation for the 103 articles with most citations is presented in Table 3.

When the areas of focus of the 103 studies with most citations related to liver transplantation in the anesthesia literature are investigated, the first three areas were transfusion practice in 17 articles (16.5\%), thromboelastography in 14 $(13.6 \%)$ and hemodynamic monitoring in 10 (9.7\%). The first four journals for the 103 articles with most citations were determined to be Anesthesia and Analgesia, British Journal of Anaesthesia, Liver Transplantation, and Anesthesiology. Of the 103 articles with most citations, 92.2\% were in SCI index journals and $7.8 \%$ were in SCI-E index journals. When the distribution of author's countries are investigated, the first 3 countries were the United States of America (USA), France and England. Of studies, $42.7 \%$ were from countries in Europe and $57.3 \%$ were from countries 
outside of Europe. There was no correlation determined between the author's continent and total number of citations. There was a significant correlation between the author's continent and mean annual citation numbers. The mean number of citations of authors from the European continent was significantly higher than authors from other continents. There was no significant correlation between the total and annual citations between journal continent and journal index. The distribution according to features of the 103 studies with most citations in the liver transplantation field in the anesthesia literature is presented in Table 4.

Table I. 103 studies with most citations related to liver transplantation in the anesthesia literature and citation rates

\begin{tabular}{|c|c|c|c|c|c|}
\hline Rank & Title & Year & Authors & $\rightarrow 0-1$ & \& 3 \\
\hline 1 & $\begin{array}{l}\text { Intraoperative Changes in Blood-Coagulation And } \\
\text { Thrombelastographic Monitoring in Liver- } \\
\text { Transplantation }\end{array}$ & 1985 & $\begin{array}{l}\text { Kang, Yg; Martin, Dj; Marquez, J; Lewis, Jh; } \\
\text { Bontempo, Fa; Shaw, Bw; Starzl, Te; Winter, } \\
\text { Pm }\end{array}$ & 456 & 13,41 \\
\hline 2 & Thrombelastography & 1992 & Mallett, Sv; Cox, Dja & 407 & 15,07 \\
\hline 3 & $\begin{array}{l}\text { Coagulation Monitoring: Current Techniques And } \\
\text { Clinical Use of Viscoelastic Point-Of-Care } \\
\text { Coagulation Devices }\end{array}$ & 2008 & Ganter, Michael T.; Hofer, Christoph K. & 376 & 34,18 \\
\hline 4 & $\begin{array}{l}\text { Early Enteral Supply of Lactobacillus And Fiber } \\
\text { Versus Selective Bowel Decontamination: A } \\
\text { Controlled Trial İn Liver Transplant Recipients }\end{array}$ & 2002 & $\begin{array}{l}\text { Rayes, N; Seehofer, D; Hansen, S; Boucsein, K; } \\
\text { Muller, Ar; Serke, S; Bengmark, S; Neuhaus, P }\end{array}$ & 279 & 16,41 \\
\hline 5 & $\begin{array}{l}\text { The Impact of Intraoperative Transfusion of } \\
\text { Platelets And Red Blood Cells On Survival After } \\
\text { Liver Transplantation }\end{array}$ & 2008 & $\begin{array}{l}\text { De Boer, Marieke T.; Christensen, Michael C.; } \\
\text { Asmussen, Mikael; Van Der Hilst, Christian S.; } \\
\text { Hendriks, Herman G. D.; Slooff, Maarten J. H.; } \\
\text { Porte, Robert J. }\end{array}$ & 165 & 15 \\
\hline 6 & $\begin{array}{l}\text { Epsilon-Aminocaproic Acid For Treatment Of } \\
\text { Fibrinolysis During Liver-Transplantation }\end{array}$ & 1987 & $\begin{array}{l}\text { Kang, Y; Lewis, Jh; Navalgund, A; Russell, Mw; } \\
\text { Bontempo, Fa; Niren, Ls; Starzl, Te }\end{array}$ & 158 & 4,94 \\
\hline 7 & $\begin{array}{llr}\text { Transfusion Triggers } & \text { In Orthotopic } & \text { Liver } \\
\text { Transplantation: A Comparison of The } & \text { Then } \\
\text { Thromboelastometry } & \text { Analyzer, } & \text { The } \\
\text { Thromboelastogram, And Conventional } \\
\text { Coagulation Tests }\end{array}$ & 2006 & $\begin{array}{l}\text { Coakley, Margaret; Reddy, Kalpana; Mackie, } \\
\text { Ian; Mallett, Susan }\end{array}$ & 141 & 10,85 \\
\hline 8 & $\begin{array}{l}\text { Effect of Low Central Venous Pressure And } \\
\text { Phlebotomy on Blood Product Transfusion } \\
\text { Requirements During Liver Transplantations }\end{array}$ & 2006 & $\begin{array}{l}\text { Massicotte, L; Lenis, S; Thibeault, L; Sassine, } \\
\text { Mp; Seal, Rf; Roy, A }\end{array}$ & 140 & 10,77 \\
\hline 9 & $\begin{array}{l}\text { Leukocyte Adhesion And Cell-Death Following } \\
\text { Orthotopic Liver-Transplantation in The Rat }\end{array}$ & 1991 & $\begin{array}{l}\text { Takei, Y; Marzi, I; Gao, Ws; Gores, Gj; } \\
\text { Lemasters, Jj; Thurman, Rg }\end{array}$ & 136 & 4,86 \\
\hline 10 & $\begin{array}{l}\text { Tranexamic Acid Reduces Blood Loss, Transfusion } \\
\text { Requirements, And Coagulation Factor Use in } \\
\text { Primary Orthotopic Liver Transplantation }\end{array}$ & 1996 & $\begin{array}{l}\text { Boylan, Jf; Klinck, Jr; Sandler, An; Arellano, R; } \\
\text { Greig, Pd; Nierenberg, H; Roger, Sl; Glynn, } \\
\text { Mfx }\end{array}$ & 126 & 5,48 \\
\hline 11 & $\begin{array}{l}\text { Evaluation of An Uncalibrated Arterial Pulse } \\
\text { Contour Cardiac Output Monitoring System in } \\
\text { Cirrhotic Patients Undergoing Liver Surgery }\end{array}$ & 2009 & $\begin{array}{l}\text { Biancofiore, G.; Critchley, L. A. H.; Lee, A.; } \\
\text { Bindi, L.; Bisa, M.; Esposito, M.; Meacci, L.; } \\
\text { Mozzo, R.; Desimone, P.; Urbani, L.; Filipponi, } \\
\text { F. }\end{array}$ & 116 & 11,6 \\
\hline 12 & $\begin{array}{l}\text { Anesthesia For Hepatic Transplantation - } \\
\text { Cardiovascular and Metabolic Alterations and } \\
\text { Their Management }\end{array}$ & 1985 & Carmichael, Fj; Lindop, Mj; Farman, Jv & 116 & 3,41 \\
\hline 13 & $\begin{array}{l}\text { Uncalibrated Pulse Contour-Derived Stroke } \\
\text { Volume Variation Predicts Fluid Responsiveness } \\
\text { in Mechanically Ventilated Patients Undergoing } \\
\text { Liver Transplantation }\end{array}$ & 2008 & $\begin{array}{l}\text { Biais, M.; Nouette-Gaulain, K.; Cottenceau, V.; } \\
\text { Revel, P.; Sztark, F. }\end{array}$ & 111 & 10,09 \\
\hline 14 & $\begin{array}{l}\text { Tranexamic Acid Reduces Red Cell Transfusion } \\
\text { Better Than Epsilon-Aminocaproic Acid or } \\
\text { Placebo in Liver Transplantation }\end{array}$ & 2000 & $\begin{array}{l}\text { Dalmau, A; Sabate, A; Acosta, F; Garcia-Huete, } \\
\text { L; Koo, M; Sansano, T; Rafecas, A; Figueras, J; } \\
\text { Jaurrieta, E; Parrilla, P }\end{array}$ & 102 & 5,37 \\
\hline
\end{tabular}




\begin{tabular}{|c|c|c|c|c|c|}
\hline 15 & \begin{tabular}{|l} 
Rotation Thromboelastometry Detects \\
Thrombocytopenia and Hypofibrinogenaemia \\
During Orthotopic Liver Transplantation
\end{tabular} & 2010 & $\begin{array}{l}\text { Roullet, S.; Pillot, J.; Freyburger, G.; Biais, M.; } \\
\text { Quinart, A.; Rault, A.; Revel, P.; Sztark, F. }\end{array}$ & 95 & 10,56 \\
\hline 16 & $\begin{array}{l}\text { Intraoperative Blood Losses and Transfusion } \\
\text { Requirements During Adult Liver Transplantation } \\
\text { Remain Difficult to Predict }\end{array}$ & 2001 & $\begin{array}{l}\text { Steib, A; Freys, G; Lehmann, C; Meyer, C; } \\
\text { Mahoudeau, G }\end{array}$ & 95 & 5,28 \\
\hline 17 & $\begin{array}{l}\text { Platelet Transfusion During Liver Transplantation } \\
\text { is Associated With Increased Postoperative } \\
\text { Mortality Due To Acute Lung Injury }\end{array}$ & 2009 & $\begin{array}{l}\text { Pereboom, Ilona T. A.; De Boer, Marieke T.; } \\
\text { Haagsma, Elizabeth B.; Hendriks, Herman G. } \\
\text { D.; Lisman, Ton; Porte, Robert J. }\end{array}$ & 92 & 9,2 \\
\hline 18 & $\begin{array}{l}\text { A Case Of Successful Enteroscopic Balloon } \\
\text { Dilation For Late Anastomotic Stricture Of } \\
\text { Choledochojejunostomy After Living Donor Liver } \\
\text { Transplantation }\end{array}$ & 2005 & $\begin{array}{l}\text { Haruta, H; Yamamoto, H; Mizuta, K; Kita, Y; } \\
\text { Uno, T; Egami, S; Hishikawa, S; Sugano, K; } \\
\text { Kawarasaki, H }\end{array}$ & 90 & 6,43 \\
\hline 19 & $\begin{array}{l}\text { Survival Rate Changes With Transfusion of Blood } \\
\text { Products During Liver Transplantation }\end{array}$ & 2005 & $\begin{array}{l}\text { Massicotte, L; Sassine, Mp; Lenis, S; Seal, Rf; } \\
\text { Roy, A }\end{array}$ & 88 & 6,29 \\
\hline 20 & $\begin{array}{l}\text { Right Heart Dysfunction, Pulmonary-Embolism, } \\
\text { And Paradoxical Embolization During Liver- } \\
\text { Transplantation - A Trans-Esophageal Two- } \\
\text { Dimensional Echocardiographic Study }\end{array}$ & 1989 & $\begin{array}{l}\text { Ellis, Je; Lichtor, Jl; Feinstein, Sb; Chung, Mr; } \\
\text { Polk, Sl; Broelsch, C; Emond, J; } \\
\text { Thistlethwaite, Jr; Roizen, Mf }\end{array}$ & 87 & 2,9 \\
\hline 21 & $\begin{array}{lll}\text { Intraoperative Fluid Management } & \text { During } \\
\text { Orthotopic Liver Transplantation } & \\
\end{array}$ & 2004 & $\begin{array}{l}\text { Schroeder, Ra; Collins, Bh; Tuttle-Newhall, E; } \\
\text { Robertson, K; Plotkin, J; Johnson, Lb; Kuo, Pc }\end{array}$ & 86 & 5,73 \\
\hline 22 & $\begin{array}{l}\text { Acute Kidney Injury During Liver Transplantation } \\
\text { As Determined By Neutrophil Gelatinase- } \\
\text { Associated Lipocalin }\end{array}$ & 2009 & $\begin{array}{l}\text { Niemann, Claus U.; Walia, Ann; Waldman, } \\
\text { Jeffrey; Davio, Michael; Roberts, John P.; } \\
\text { Hirose, Ryutaro; Feiner, John }\end{array}$ & 85 & 8,5 \\
\hline 23 & $\begin{array}{l}\text { Extrahepatic Metabolısm of Propofol In Man } \\
\text { During The Anhepatıc Phase of Orthotopic Liver- } \\
\text { Transplantation }\end{array}$ & 1992 & $\begin{array}{l}\text { Veroli, P; Okelly, B; Bertrand, F; Trouvin, Jh; } \\
\text { Farinotti, R; Ecoffey, C }\end{array}$ & 85 & 3,15 \\
\hline 24 & Transfusion Predictors in Liver Transplant & 2004 & Massicotte, L; Sassine, Mp; Lenis, S; Roy, A & 84 & 5,6 \\
\hline 25 & $\begin{array}{l}\text { Portopulmonary Hypertension and The Liver } \\
\text { Transplant Candidate }\end{array}$ & 1999 & $\begin{array}{l}\text { Kuo, Pc; Plotkin, Js; Gaine, S; Schroeder, Ra; } \\
\text { Rustgi, Vk; Rubin, Lj; Johnson, Lb }\end{array}$ & 84 & 4,2 \\
\hline 26 & $\begin{array}{l}\text { Institutional Variability in Transfusion Practice } \\
\text { For Liver Transplantation }\end{array}$ & 2003 & Ozier, Y; Pessione, F; Samain, E; Courtois, F & 83 & 5,19 \\
\hline 27 & $\begin{array}{l}\text { Coagulation Defects Do Not Predict Blood Product } \\
\text { Requirements During Liver Transplantation }\end{array}$ & 2008 & $\begin{array}{l}\text { Massicotte, Luc; Beaulieu, } \quad \text { Danielle; } \\
\text { Thibeault, Lynda; Roy, Jean-Denis; Marleau, } \\
\text { Denis; Lapointe, Real; Roy, Andre } \\
\end{array}$ & 82 & 7,45 \\
\hline 28 & $\begin{array}{l}\text { History of Pediatric Liver Transplantation. Where } \\
\text { Are We Coming From? Where Do We Stand? }\end{array}$ & 2002 & 管 & 82 & 4,82 \\
\hline 29 & $\begin{array}{l}\text { Cardiovascular Depression Secondary to Ionic } \\
\text { Hypocalcemia During Hepatic Transplantation in } \\
\text { Humans }\end{array}$ & 1986 & $\begin{array}{l}\text { Marquez, J; Martin, D; Virji, Ma; Kang, Yg; } \\
\text { Warty, Vs; Shaw, B; Sassano, Jj; Waterman, P; } \\
\text { Winter, Pm; Pinsky, Mr }\end{array}$ & 81 & 2,45 \\
\hline 30 & $\begin{array}{l}\text { Organ Transplantation: Historical Perspective and } \\
\text { Current Practice }\end{array}$ & 2012 & Watson, C. J. E.; Dark, J. H. & 75 & 10,71 \\
\hline 31 & $\begin{array}{l}\text { Use of Heparinase Modified Thrombelastography } \\
\text { in Liver Transplantation }\end{array}$ & 1997 & Harding, Sa; Mallett, Sv; Peachey, Td; Cox, Dj & 71 & 3,23 \\
\hline 32 & $\begin{array}{l}\text { Moderate Primary Pulmonary Hypertension in } \\
\text { Patients Undergoing Liver Transplantation }\end{array}$ & 1996 & $\begin{array}{l}\text { Taura, P; Garciavaldecasas, Jc; Beltran, J; } \\
\text { Izquierdo, E; Navasa, M; Salablanch, J; Mas, A; } \\
\text { Balust, J; Grande, L; Visa, J }\end{array}$ & 69 & 3 \\
\hline 33 & $\begin{array}{l}\text { Fast Interpretation of Thromboelastometry in } \\
\text { Non-Cardiac Surgery: Reliability in Patients With } \\
\text { Hypo-, Normo-, And Hypercoagulability }\end{array}$ & 2013 & $\begin{array}{l}\text { Goerlinger, K.; Dirkmann, D.; Solomon, C.; } \\
\text { Hanke, A. A. }\end{array}$ & 68 & 11,33 \\
\hline 34 & $\begin{array}{l}\text { Air-Embolism Associated With Venovenous } \\
\text { Bypass During Orthotopic Liver-Transplantation }\end{array}$ & 1987 & $\begin{array}{llll}\text { Khoury, Gf; } & \text { Mann, } \quad \text { Me; } & \text { Porot, } & \text { Mj; } \\
\text { Abdulrasool, Ih; } & \text { Busuttil, Rw } & & \\
\end{array}$ & 68 & 2,13 \\
\hline 35 & $\begin{array}{l}\text { Quantitative Measurement of } \\
\text { Thromboelastography As A Function of Platelet } \\
\text { Count }\end{array}$ & 1999 & Oshita, K; Az-Ma, T; Osawa, Y; Yuge, O & 65 & 3,25 \\
\hline 36 & $\begin{array}{l}\text { Perioperative Care of The Liver-Transplant } \\
\text { Patient. Part } 2 .\end{array}$ & 1994 & $\begin{array}{l}\text { Carton, Eg; Plevak, Dj; Kranner, Pw; Rettke, } \\
\text { Sr; Geiger, Hj; Coursin, Db }\end{array}$ & 65 & 2,6 \\
\hline 37 & $\begin{array}{l}\text { Right Ventricular-Function During Orthotopic } \\
\text { Liver-Transplantation }\end{array}$ & 1993 & $\begin{array}{l}\text { Dewolf, Am; Begliomini, B; Gasior, Ta; Kang, } \\
\text { Y; Pinsky, Mr }\end{array}$ & 65 & 2,5 \\
\hline
\end{tabular}




\begin{tabular}{|c|c|c|c|c|c|}
\hline 38 & $\begin{array}{l}\text { Acute Kidney Injury Following Orthotopic Liver } \\
\text { Transplantation: Incidence, Risk Factors, and } \\
\text { Effects on Patient and Graft Outcomes }\end{array}$ & 2015 & $\begin{array}{l}\text { Hilmi, I. A.; Damian, D.; Al-Khafaji, A.; } \\
\text { Planinsic, R.; Boucek, C.; Sakai, T.; Chang, C. - } \\
\text { C. H.; Kellum, J. A. }\end{array}$ & 62 & 15,5 \\
\hline 39 & $\begin{array}{l}\text { Cardiac Output Measurement in Patients } \\
\text { Undergoing Liver Transplantation: Pulmonary } \\
\text { Artery Catheter Versus Uncalibrated Arterial } \\
\text { Pressure Waveform Analysis } \\
\end{array}$ & 2008 & $\begin{array}{l}\text { Matthieu, Biais; Karine, Nouette-Gaulain; } \\
\text { Vincent, Cottenceau; Alain, Vallet; Francois, } \\
\text { Cochard Jean; Philippe, Revel; Francois, } \\
\text { Sztark }\end{array}$ & 60 & 5,45 \\
\hline 40 & $\begin{array}{l}\text { Effect of Intraoperative Low-Dose Dopamine on } \\
\text { Renal-Function In Liver-Transplant Recipients }\end{array}$ & 1991 & 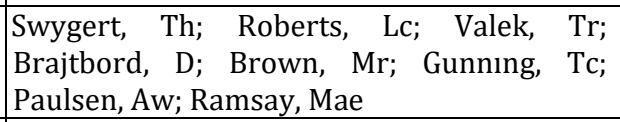 & 59 & 2,11 \\
\hline 41 & $\begin{array}{l}\text { Transfusion Rate For } 500 \text { Consecutive } \\
\text { Transplantations: Experience of } \\
\text { Transplantation Center }\end{array}$ & 2012 & $\begin{array}{l}\text { Massicotte, Luc; Denault, Andre Y.; Beaulieu, } \\
\text { Danielle; Thibeault, Lynda; Hevesi, Zoltan; } \\
\text { Nozza, Anna; Lapointe, Real; Roy, Andre }\end{array}$ & 57 & 8,14 \\
\hline 42 & $\begin{array}{l}\text { Evaluation of A New Software Version of The } \\
\text { Flotrac/Vigileo (Version 3.02) and A Comparison } \\
\text { With Previous Data in Cirrhotic Patients } \\
\text { Undergoing Liver Transplant Surgery }\end{array}$ & 2011 & $\begin{array}{l}\text { Biancofiore, Gianni; Critchley, Lester A. H.; } \\
\text { Lee, Anna; Yang, Xiao-Xing; Bindi, Lucia M.; } \\
\text { Esposito, Massimo; Bisa, Massimo; Meacci, } \\
\text { Luca; Mozzo, Roberto; Filipponi, Franco }\end{array}$ & 57 & 7,13 \\
\hline 43 & $\begin{array}{l}\text { Pharmacokinetics and Pharmacodynamics of } \\
\text { Cisatracurium in Patients With End-Stage Liver } \\
\text { Disease Undergoing Liver Transplantation }\end{array}$ & 1996 & $\begin{array}{l}\text { Dewolf, Am; Freeman, Ja; Scott, Vl; Tullock, } \\
\text { W; Smith, Da; Kisor, Df; Kerls, S; Cook, Dr }\end{array}$ & 56 & 2,43 \\
\hline 44 & $\begin{array}{lll}\begin{array}{l}\text { Pulmonary-Hypertension } \\
\text { Transplantation }\end{array} & \text { and Liver- } \\
\end{array}$ & 1993 & Dewolf, Am; Scott, Vl; Gasıor, T; Kang, Yg & 56 & 2,15 \\
\hline 45 & $\begin{array}{l}\text { Massive Pulmonary Thromboembolism During } \\
\text { Liver-Transplantation }\end{array}$ & 1988 & $\begin{array}{l}\text { Navalgund, Aa; Kang, Yg; Sarner, Jb; Jahr, Js; } \\
\text { Gieraerts, R }\end{array}$ & 56 & 1,81 \\
\hline 46 & $\begin{array}{l}\text { Poor Prediction of Blood Transfusion } \\
\text { Requirements in Adult Liver Transplantations } \\
\text { From Preoperative Variables }\end{array}$ & 2000 & Findlay, Jy; Rettke, Sr & 55 & 2,89 \\
\hline 47 & $\begin{array}{|lc|}\text { Intraoperative } & \text { Transesophageal } \\
\text { Echocardiography During Liver Transplantation }\end{array}$ & 1996 & $\begin{array}{l}\begin{array}{l}\text { Suriani, Rj; } \\
\text { Konstadt, } \mathrm{S}\end{array} \\
\end{array}$ & 55 & 2,39 \\
\hline 48 & $\begin{array}{|lccr|}\text { Continuous } & \text { Small-Dose } & \text { Aprotinin } & \text { Controls } \\
\text { Fibrinolysis } & \text { During } & \text { Orthotopic } & \text { Liver } \\
\text { Transplantation } & & & \\
\end{array}$ & 1996 & $\begin{array}{l}\text { Marcel, Rj; Stegall, Wc; Suit, Ct; Arnold, Jc; } \\
\text { Vera, Rl; Ramsay, Mae; Odonnell, Mb; } \\
\text { Swygert, Th; Hein, Hat; Whitten, Cw }\end{array}$ & 55 & 2,39 \\
\hline 49 & $\begin{array}{l}\text { lulmonary-Hypertension Associated With Liver- } \\
\text { Pisease is not Reversible After Liver- } \\
\text { Transplantation }\end{array}$ & 1992 & $\begin{array}{l}\text { Prager, Mc; Cauldwell, Ca; Ascher, Nl; } \\
\text { Roberts, Jp; Wolfe, Cl }\end{array}$ & 55 & 2,04 \\
\hline 50 & $\begin{array}{l}\text { Increased Prothrombin Time and Platelet Counts } \\
\text { in Living Donor Right Hepatectomy: Implications } \\
\text { For Epidural Anesthesia }\end{array}$ & 2004 & $\begin{array}{l}\text { Siniscalchi, A; Begliomini, B; De Pietri, L; } \\
\text { Braglia, V; Gazzi, M; Masetti, M; Di Benedetto, } \\
\text { F; Pinna, Ad; Miller, Cm; Pasetto, A }\end{array}$ & 54 & 3,6 \\
\hline 51 & $\begin{array}{l}\text { The Effect of Methylene Blue on The } \\
\text { Hemodynamic Changes } \\
\begin{array}{l}\text { Reperfusion Injury in } \\
\text { Tring }\end{array} \\
\text { Transplantation }\end{array}$ & 2002 & $\begin{array}{l}\text { Koelzow, H; Gedney, Ja; Baumann, J; Snook, } \\
\text { Nj; Bellamy, Mc }\end{array}$ & 54 & 3,18 \\
\hline 52 & $\begin{array}{l}\text { Monitoring Patients at Risk of Massive } \\
\text { Transfusion With Thrombelastography or } \\
\text { Thromboelastometry: A Systematic Review }\end{array}$ & 2011 & $\begin{array}{l}\text { Wikkelsoe, A. J.; Afshari, A.; Wetterslev, J.; } \\
\text { Brok, J.; Moeller, A. M. }\end{array}$ & 53 & 6,63 \\
\hline 53 & $\begin{array}{l}\text { Predictors of Blood Product Use in Orthotopic } \\
\text { Liver Transplantation Using The Piggyback } \\
\text { Hepatectomy Technique }\end{array}$ & 2007 & $\begin{array}{l}\text { Mangus, R. S.; Kinsella, S. B.; Nobari, M. M.; } \\
\text { Fridell, J. A.; Vianna, R. M.; Ward, E. S.; Nobari, } \\
\text { R.; Tector, A. J. }\end{array}$ & 53 & 4,42 \\
\hline 54 & $\begin{array}{l}\text { Outcome of Liver Transplantation For Patients } \\
\text { with Pulmonary Hypertension }\end{array}$ & 2002 & Starkel, P; Vera, A; Gunson, B; Mutimer, D & 53 & 3,12 \\
\hline 55 & $\begin{array}{l}\text { Pulmonary-Artery Hypertension Complicating } \\
\text { Anesthesia For Liver-Transplantation }\end{array}$ & 1992 & Cheng, Ey; Woehlck, Hj & 53 & 1,96 \\
\hline 56 & $\begin{array}{l}\text { Considerations For Anesthetic Management } \\
\text { During Veno-Venous Bypass in Adult Hepatic } \\
\text { Transplantation }\end{array}$ & 1989 & $\begin{array}{l}\text { Paulsen, Aw; Whitten, Cw; Ramsay, Mae; } \\
\text { Klintmalm, Gb }\end{array}$ & 53 & 1,77 \\
\hline 57 & $\begin{array}{|llr|}\begin{array}{l}\text { Anesthetic Management } \\
\text { Transplantation }\end{array} & \text { of } & \text { Hepatic } \\
\end{array}$ & 2008 & Ozier, Yves; Klinck, John R. & 52 & 4,73 \\
\hline 58 & $\begin{array}{l}\text { Intraoperative Resource Utilization in Anesthesia } \\
\text { For Liver Transplantation in The United States: A } \\
\text { Survey }\end{array}$ & 2003 & Schumann, R & 52 & 3,25 \\
\hline
\end{tabular}




\begin{tabular}{|c|c|c|c|c|c|}
\hline 59 & \begin{tabular}{|l} 
Endogenous \\
Significantly Impair Coagulation in Patients \\
Undergoing Orthotopic Liver Transplantation
\end{tabular} & 1998 & $\begin{array}{l}\text { Kettner, Sc; Gonano, C; Seebach, F; Sitzwohl, } \\
\text { C; Acimovic, S; Stark, J; Schellongowski, A; } \\
\text { Blaicher, A; Felfernig, M; Zimpfer, M }\end{array}$ & 52 & 2,48 \\
\hline 60 & $\begin{array}{l}\text { Continuous Small-Dose Tranexamic Acid Reduces } \\
\text { Fibrinolysis But Not Transfusion Requirements } \\
\text { During Orthotopic Liver Transplantation }\end{array}$ & 1997 & $\begin{array}{l}\text { Kaspar, M; Ramsay, Mae; Nguyen, At; } \\
\text { Cogswell, M; Hurst, G; Ramsay, Kj }\end{array}$ & 52 & 2,36 \\
\hline 61 & Liver Transplantation in The Morbidly Obese & 1996 & $\begin{array}{l}\text { Braunfeld, My; Chan, S; Pregler, J; Neelakanta, } \\
\text { G; Sopher, Mj; Busuttil, Rw; Csete, M }\end{array}$ & 52 & 2,26 \\
\hline 62 & $\begin{array}{l}\text { Arginase Release Following Liver Reperfusion - } \\
\text { Evidence of Hemodynamic Action of Arginase } \\
\text { Infusions }\end{array}$ & 1995 & $\begin{array}{l}\text { Langle, F; Roth, E; Steininger, R; Winkler, S; } \\
\text { Muhlbacher, F }\end{array}$ & 52 & 2,17 \\
\hline 63 & $\begin{array}{l}\text { Association of Intraoperative Hypotension and } \\
\text { Pulmonary Hypertension With Adverse Outcomes } \\
\text { After Orthotopic Liver Transplantation }\end{array}$ & 2003 & $\begin{array}{l}\text { Reich, Dl; Wood, Rk; Emre, S; Bodian, Ca; } \\
\text { Hossain, S; Krol, M; Feierman, D }\end{array}$ & 51 & 3,19 \\
\hline 64 & $\begin{array}{l}\text { Postoperative Tracheal Extubation After } \\
\text { Orthotopic Liver Transplantation }\end{array}$ & 2001 & $\begin{array}{l}\text { Glanemann, M; Langrehr, J; Kaisers, U; } \\
\text { Schenk, R; Muller, A; Stange, B; Neumann, U; } \\
\text { Bechstein, Wo; Falke, K; Neuhaus, P }\end{array}$ & 51 & 2,83 \\
\hline 65 & $\begin{array}{l}\text { Immediate Tracheal Extubation After Liver } \\
\text { Transplantation: Experience of Two Transplant } \\
\text { Centers }\end{array}$ & 1997 & Mandell, Ms; Lockrem, J; Kelley, Sd & 51 & 2,32 \\
\hline 66 & $\begin{array}{l}\text { Extended Donor Criteria Have No Negative Impact } \\
\text { on Early Outcome After Liver Transplantation: A } \\
\text { Single-Center Multivariate Analysis }\end{array}$ & 2007 & $\begin{array}{l}\text { Schemmer, P.; Nickkholgh, A.; Hinz, U.; } \\
\text { Gerling, T.; Mehrabi, A.; Sauer, P.; Encke, J.; } \\
\text { Friess, H.; Weitz, J.; Buechler, M. W.; Schmidt, } \\
\text { J. }\end{array}$ & 50 & 4,17 \\
\hline 67 & $\begin{array}{l}\text { Altered Hematologic Profiles Following Donor } \\
\text { Right Hepatectomy and Implications For } \\
\text { Perioperative Analgesic Management }\end{array}$ & 2004 & $\begin{array}{l}\text { Schumann, R; Zabala, L; Angelis, M; Bonney, } \\
\text { I; Tighiouart, H; Carr, Db }\end{array}$ & 50 & 3,33 \\
\hline 68 & $\begin{array}{l}\text { Systematic Grading of Surgical Complications in } \\
\text { Live Liver Donors According to Clavien's System }\end{array}$ & 2006 & $\begin{array}{l}\text { Tamura, Sumihito; Sugawara, Yasuhiko; } \\
\text { Kaneko, Junichi; Yamashiki, Noriyo; Kishi, } \\
\text { Yoji; Matsui, Yuichi; Kokudo, Norihiro; } \\
\text { Makuuchi, Masatoshi }\end{array}$ & 49 & 3,77 \\
\hline 69 & $\begin{array}{l}\text { Comparison of Invasive and Noninvasive } \\
\text { Measurement of Plasma Disamearance Rate of } \\
\text { Indocyanine Green in Patients Undergoing Liver } \\
\text { Transplantation: A Prospective Investigator- } \\
\text { Blinded Study }\end{array}$ & 2004 & $\begin{array}{l}\text { Faybik, P; Krenn, Cg; Baker, A; Lahner, D; } \\
\text { Berlakovich, G; Steltzer, H; Hetz, H }\end{array}$ & 49 & 3,27 \\
\hline 70 & $\begin{array}{l}\text { Preload And Haemodynamic Assessment During } \\
\text { Liver Transplantation: A Comparison Between } \\
\text { The Pulmonary Artery Catheter and } \\
\text { Transpulmonary Indicator Dilution Techniques }\end{array}$ & 2002 & $\begin{array}{l}\text { Della Rocca, G; Costa, Mg; Coccia, C; Pompei, } \\
\text { L; Pietropaoli, P }\end{array}$ & 49 & 2,88 \\
\hline 71 & $\begin{array}{l}\text { Extrahepatic Morphine-Metabolism in Man } \\
\text { During The Anhepatic Phase of Orthotopic Liver- } \\
\text { Transplantation }\end{array}$ & 1989 & Bodenham, A; Quinn, K; Park, Gr & 49 & 1,63 \\
\hline 72 & $\begin{array}{l}\text { Predictors of Hyperkalemia in The } \\
\text { Prereperfusion, Early Postreperfusion, and Late } \\
\text { Postreperfusion Periods During Adult Liver } \\
\text { Transplantation }\end{array}$ & 2007 & $\begin{array}{l}\text { Xia, Victor W.; Ghobrial, Rafik M.; Du, Bin; } \\
\text { Chen, Tabitha; Hu, Ke-Qin; Hiatt, Jonathan R.; } \\
\text { Busuttil, Ronald W.; Steadman, Randolph H. }\end{array}$ & 47 & 3,92 \\
\hline 73 & $\begin{array}{l}\text { Fibrinolysis During Liver Transplantation is } \\
\text { Enhanced By Using Solvent/Detergent Virus- } \\
\text { Inactivated Plasma (Esdep (R)) }\end{array}$ & 2002 & $\begin{array}{l}\text { De Jonge, J; Groenland, Thn; Metselaar, Hj; } \\
\text { Ijzermans, Jnm; Van Vliet, Hhdm; Visser, L; } \\
\text { Tilanus, Hw }\end{array}$ & 47 & 2,76 \\
\hline 74 & \begin{tabular}{|l} 
Does Adult Liver-Transplantation Without \\
Venovenous Bypass Result in Renal-Failure
\end{tabular} & 1992 & Veroli, P; Elhage, C; Ecoffey, C & 47 & 1,74 \\
\hline 75 & $\begin{array}{l}\text { Pulmonary Thromboembolism During Adult Liver } \\
\text { Transplantation: Incidence, Clinical Presentation, } \\
\text { Outcome, Risk Factors, and Diagnostic Predictors }\end{array}$ & 2012 & $\begin{array}{l}\text { Sakai, T.; Matsusaki, T.; Dai, F.; Tanaka, K. A.; } \\
\text { Donaldson, J. B.; Hilmi, I. A.; Marsh, J. Wallis; } \\
\text { Planinsic, R. M.; Humar, A. }\end{array}$ & 46 & 6,57 \\
\hline 76 & $\begin{array}{l}\text { Monitoring of Haemostasis in Liver } \\
\text { Transplantation: Comparison of } \\
\text { Based and Point of Care Tests }\end{array}$ & 2010 & $\begin{array}{l}\text { Herbstreit, F.; Winter, E. M.; Peters, J.; } \\
\text { Hartmann, M. }\end{array}$ & 46 & 5,11 \\
\hline 77 & $\begin{array}{l}\text { Patterns of Coagulopathy During Liver- } \\
\text { Transplantation - Experience With The First } 75 \\
\text { Cases Using Thrombelastography }\end{array}$ & 1994 & $\begin{array}{l}\text { Mcnicol, Pl; Liu, G; Harley, Id; Mccall, Pr; } \\
\text { Przybylowski, Gm; Bowkett, J; Angus, Pw; } \\
\text { Hardy, Kj; Jones, Rm }\end{array}$ & 46 & 1,84 \\
\hline
\end{tabular}




\begin{tabular}{|c|c|c|c|c|c|}
\hline 78 & $\begin{array}{l}\text { Epidural Catheter and Increased Prothrombin } \\
\text { Time After Right Lobe Hepatectomy For Living } \\
\text { Donor Transplantation }\end{array}$ & 2000 & Borromeo, Cj; Stix, Ms; Lally, A; Pomfret, Ea & 45 & 2,37 \\
\hline 79 & $\begin{array}{l}\text { Temperature Corrected Thrombelastography in } \\
\text { Hypothermic Patients }\end{array}$ & 1995 & $\begin{array}{l}\text { Douning, Lk; Ramsay, Mae; Swygert, Th; } \\
\text { Hicks, Kn; Hein, Hat; Gunning, Tc; Suit, Ct }\end{array}$ & 45 & 1,88 \\
\hline 80 & $\begin{array}{l}\text { Three Patients Requiring Both Coronary-Artery } \\
\begin{array}{ll}\text { Bypass-Surgery and Orthotopic Liver- } \\
\text { Transplantation }\end{array} \\
\end{array}$ & 1995 & $\begin{array}{l}\text { Morris, Jj; Hellman, Cl; Gawey, Bj; Ramsay, } \\
\text { Mae; Valek, Tr; Gunning, Tc; Swygert, Th; } \\
\text { Shorelesserson, L; Lalehzarian, F; Brayman, } \\
\text { Kl; Brennan, Ta }\end{array}$ & 45 & 1,88 \\
\hline 81 & $\begin{array}{|lccc|}\begin{array}{l}\text { Anesthesia } \\
\text { Transplantation }\end{array} & \text { Pedıatrıc } & \text { Orthotopic Liver- } \\
\end{array}$ & 1985 & Borland, Lm; Roule, M; Cook, Dr & 45 & 1,32 \\
\hline 82 & $\begin{array}{l}\text { Meld Score And Blood Product Requirements } \\
\text { During Liver Transplantation: No Link }\end{array}$ & 2009 & $\begin{array}{l}\text { Massicotte, Luc; Beaulieu, Danielle; Roy, } \\
\text { Jean-Denis; Marleau, Denis; Vandenbroucke, } \\
\text { Frank; Dagenais, Michel; Lapointe, Real; Roy, } \\
\text { Andre }\end{array}$ & 43 & 4,3 \\
\hline 83 & \begin{tabular}{|lrrr}
$\begin{array}{l}\text { Fatal Pulmonary } \\
\text { Transplantation }\end{array}$ & Embolism & During & Liver \\
\end{tabular} & 1997 & $\begin{array}{l}\text { Sopher, M; Braunfeld, M; Shackleton, C; } \\
\text { Busuttil, Rw; Sangwan, S; Csete, M }\end{array}$ & 43 & 1,95 \\
\hline 84 & $\begin{array}{l}\text { Continuous Versus Intermittent Thermodilution } \\
\text { Cardiac Output Measurement During Orthotopic } \\
\text { Liver Transplantation }\end{array}$ & 1997 & $\begin{array}{l}\text { Bottiger, Bw; Sinner, B; Motsch, J; Bach, A; } \\
\text { Bauer, H; Martin, E }\end{array}$ & 43 & 1,95 \\
\hline 85 & $\begin{array}{l}\text { Amelioration of Lactic-Acidosis With } \\
\text { Dichloroacetate During Liver-Transplantation in } \\
\text { Humans }\end{array}$ & 1994 & $\begin{array}{l}\text { Shangraw, Re; Winter, R; Hromco, J; } \\
\text { Robinson, St; Gallaher, Ej }\end{array}$ & 43 & 1,72 \\
\hline 86 & $\begin{array}{l}\text { Systematic Review And Meta-Analysis of The } \\
\text { Impact of Computed Tomography-Assessed } \\
\text { Skeletal Muscle Mass on Outcome in Patients } \\
\text { Awaiting or Undergoing Liver Transplantation }\end{array}$ & 2016 & $\begin{array}{l}\text { Van Vugt, J. L. A.; Levolger, S.; De Bruin, R. W. } \\
\text { F.; Van Rosmalen, J.; Metselaar, H. J.; } \\
\text { Ijzermans, J. N. M. }\end{array}$ & 42 & 14 \\
\hline 87 & $\begin{array}{l}\text { A Comparison of Stroke Volume Variation } \\
\text { Measured By Vigileo (Tm)/Flotrac (Tm) System } \\
\text { and Aortic Doppler Echocardiography }\end{array}$ & 2009 & $\begin{array}{l}\text { Biais, Matthieu; Nouette-Gaulain, Karine; } \\
\text { Roullet, Stephanie; Quinart, Alice; Revel, } \\
\text { Philippe; Sztark, Francois }\end{array}$ & 41 & 4,1 \\
\hline 88 & \begin{tabular}{|lcc} 
Four Cases of & Cardiopulmonary \\
Thromboembolism During Liver Transplantation \\
Without The Use of Antifibrinolytic Drugs
\end{tabular} & 2005 & $\begin{array}{l}\text { Lerner, Ab; Sundar, E; Mahmood, F; Sarge, T; } \\
\text { Hanto, Dw; Panzica, Pj }\end{array}$ & 41 & 2,93 \\
\hline 89 & $\begin{array}{l}\text { Fast Track in Liver Transplantation: } 5 \text { Years' } \\
\text { Experience }\end{array}$ & 2005 & $\begin{array}{l}\text { Biancofiore, G; Bindi, Ml; Romanelli, Am; } \\
\text { Boldrini, A; Bisa, M; Esposito, M; Urbani, L; } \\
\text { Catalano, G; Mosca, F; Filipponi, E }\end{array}$ & 41 & 2,93 \\
\hline 90 & $\begin{array}{l}\text { Transesophageal Echocardiography Utilization in } \\
\text { High-Volume Liver Transplantation Centers in } \\
\text { The United States }\end{array}$ & 2008 & $\begin{array}{l}\text { Wax, David B.; Torres, Antonio; Scher, Corey; } \\
\text { Leibowitz, Andrew B. }\end{array}$ & 40 & 3,64 \\
\hline 91 & $\begin{array}{l}\text { Regional Cerebral Oxygen Saturation is A } \\
\text { Sensitive Marker of Cerebral Hypoperfusion } \\
\text { During Orthotopic Liver Transplantation }\end{array}$ & 2004 & $\begin{array}{l}\text { Plachky, J; Hofer, S; Volkmann, M; Martin, E; } \\
\text { Bardenheuer, Hj; Weigand, Ma }\end{array}$ & 40 & 2,67 \\
\hline 92 & $\begin{array}{l}\text { Clinical Predictors of Pulmonary Hypertension in } \\
\text { Patients Undergoing Liver Transplant Evaluation }\end{array}$ & 2000 & $\begin{array}{l}\text { Pilatis, Nd; Jacobs, Le; Rerkpattanapipat, P; } \\
\text { Kotler, Mn; Owen, A; Manzarbeitia, C; Reich, } \\
\text { D; Rothstein, K; Munoz, Sj }\end{array}$ & 40 & 2,11 \\
\hline 93 & $\begin{array}{l}\text { Pulmonary Thromboembolism During } \text { Liver } \\
\text { Transplantation: Possible Association With } \\
\text { Antifibrinolytic Drugs and Novel Treatment } \\
\text { Options }\end{array}$ & 2000 & $\begin{array}{l}\text { O'connor, Cj; Roozeboom, D; Brown, R; } \\
\text { Tuman, Kj }\end{array}$ & 39 & 2,05 \\
\hline 94 & $\begin{array}{l}\begin{array}{l}\text { Predictive Factors of Hyperfibrinolytic Activity } \\
\text { During Liver-Transplantation in Cirrhotic- } \\
\text { Patients }\end{array} \\
\end{array}$ & 1994 & $\begin{array}{l}\text { Steib, A; Gengenwin, N; Freys, G; Boudjema, } \\
\text { K; Levy, S; Otteni, Jc }\end{array}$ & 39 & 1,56 \\
\hline 95 & \begin{tabular}{|l} 
Aprotinin Administration and Pulmonary \\
Thromboembolism During Orthotopic Liver \\
Transplantation: Report of Two Cases
\end{tabular} & 2001 & Fitzsimons, Mg; Peterfreund, Ra; Raines, De & 38 & 2,11 \\
\hline 96 & $\begin{array}{l}\text { Pharmacokinetics of Morphine in Two Children } \\
\text { Before and After Liver-Transplantation }\end{array}$ & 1986 & Shelly, Mp; Cory, Ep; Park, Gr & 38 & 1,15 \\
\hline
\end{tabular}




\begin{tabular}{|c|c|c|c|c|c|}
\hline 97 & \multicolumn{4}{|c|}{$\begin{array}{l}\text { The Safety of Modern Hydroxyethyl Starch in } \\
\text { Living Donor Liver Transplantation: A } \\
\text { Comparison With Human Albumin }\end{array}$} & 200 \\
\hline 98 & \multicolumn{4}{|c|}{$\begin{array}{l}\text { Association Between Central Venous Pressure and } \\
\text { Blood Loss During Hepatic Resection in } 984 \\
\text { Living Donors }\end{array}$} & 200 \\
\hline 99 & \multicolumn{4}{|c|}{$\begin{array}{l}\text { Thromboelastometry For Guiding Bleeding } \\
\text { Management of The Critically Ill Patient: A } \\
\text { Systematic Review of The Literature }\end{array}$} & 2014 \\
\hline 100 & \multicolumn{4}{|c|}{$\begin{array}{l}\text { Anesthesia Care For Adult Live Donor } \\
\text { Hepatectomy: Our Experiences With } 100 \text { Cases }\end{array}$} & 2007 \\
\hline 101 & \multicolumn{4}{|c|}{$\begin{array}{l}\text { A Matched Comparison Study of Medical and } \\
\text { Psychiatric Complications and Anesthesia and } \\
\text { Analgesia Requirements in Methadone- } \\
\text { Maintained Liver Transplant Recipients }\end{array}$} & 200 \\
\hline 102 & \multicolumn{4}{|c|}{$\begin{array}{l}\text { Blood-Transfusion Requirements and Blood } \\
\text { Salvage in Donors Undergoing Right Hepatectomy } \\
\text { For Living Related Liver Transplantation }\end{array}$} & 2003 \\
\hline 103 & \multicolumn{4}{|c|}{\begin{tabular}{|l} 
Clinical-Evaluation of Autotransfusion During \\
Liver-Transplantation
\end{tabular}} & 199 \\
\hline \multicolumn{6}{|c|}{$\begin{array}{l}\text { Table II. Distribution of first authors for } 103 \text { studies with most } \\
\text { citations related to liver transplantation in the anesthesia } \\
\text { literature }\end{array}$} \\
\hline \multicolumn{2}{|c|}{ First Author } & $\%$ & First Author & $\mathbf{n}$ & $\%$ \\
\hline \multicolumn{2}{|c|}{ Massicotte, $\mathrm{L}$} & 5.8 & Lerner, $\mathrm{AB}$ & 1 & 1.0 \\
\hline \multicolumn{2}{|l|}{ Kang, Y } & 2.9 & Lutz, JT & 1 & 1.0 \\
\hline \multicolumn{2}{|c|}{ Dewolf, Am } & 2.9 & Mallett, Sv & 1 & 1.0 \\
\hline \multicolumn{2}{|c|}{ Biancofiore, G } & 2.9 & Mandell, MS & 1 & 1.0 \\
\hline \multicolumn{2}{|l|}{ Biais, M } & 1.9 & Mangus, R.S. & 1 & 1.0 \\
\hline \multicolumn{2}{|l|}{ Ozier, Y } & 1.9 & Marcel, RJ & 1 & 1.0 \\
\hline \multicolumn{2}{|c|}{ Schumann, R } & 1.9 & Marquez, J & 1 & 1.0 \\
\hline \multicolumn{2}{|l|}{ Steib, A } & 1.9 & Matthieu, Biais & 1 & 1.0 \\
\hline \multicolumn{2}{|l|}{ Veroll, $\mathbf{P}$} & 1.9 & Mcnıcol, Pl & 1 & 1.0 \\
\hline \multicolumn{2}{|c|}{ Bodenham, A } & 1.0 & Morris, Jj & 1 & 1.0 \\
\hline \multicolumn{2}{|c|}{ Borland, Lm } & 1.0 & Mukhtar, Ahmed & 1 & 1.0 \\
\hline \multicolumn{2}{|c|}{ Borromeo, CJ } & 1.0 & Navalgund, Aa & 1 & 1.0 \\
\hline \multicolumn{2}{|c|}{ Bottiger. BW } & 1.0 & Niemann, Claus U & 1 & 1.0 \\
\hline \multicolumn{2}{|c|}{ Boylan, JF } & 1.0 & O'Connor, CJ & 1 & 1.0 \\
\hline \multicolumn{2}{|c|}{ Braunfeld, MY } & 1.0 & Oshita, $\mathrm{K}$ & 1 & 1.0 \\
\hline \multicolumn{2}{|c|}{ Carmıchael, Fj } & 1.0 & Otte, JB & 1 & 1.0 \\
\hline \multicolumn{2}{|c|}{ Carton, Eg } & 1.0 & Paulsen, Aw & 1 & 1.0 \\
\hline \multicolumn{2}{|c|}{ Cheng, Ey } & 1.0 & Pereboom, Ilonat.A & 1 & 1.0 \\
\hline \multicolumn{2}{|c|}{ Chhibber, Ashwani } & 1.0 & Pilatis, ND & 1 & 1.0 \\
\hline \multicolumn{2}{|c|}{\begin{tabular}{|l|} 
Coakley, Margaret \\
\end{tabular}} & 1.0 & Plachky, J & 1 & 1.0 \\
\hline \multicolumn{2}{|c|}{ Dalmau, A } & 1.0 & Prager, Mc & 1 & 1.0 \\
\hline
\end{tabular}

Mukhtar, Ahmed; Aboulfetouh, Fawzia; Obayah, Gihan; Salah, Maged; Emam, Mohamed; Khater, Yehia; Akram, Ramzia; Hoballah, Aly; Bahaa, Mohamed; Elmeteini, Mahmoud; Hamza, Alaa

Kim, Y. K.; Chin, J. H.; Kang, S. J.; Jun, I. G.; Song, J. G.; Jeong, S. M.; Park, J. Y.; Hwang, G. S.

Haas, T.; Goerlinger, K.; Grassetto, A.; Agostini, V.; Simioni, P.; Nardi, G.; Ranucci, M.

Chhibber, Ashwani; Dziak, Jason; Kolano, Jefferey; Norton, J. Russell; Lustik, Stewart

Weinrieb, Rm; Barnett, R; Lynch, Kg; Depiano, M; Atanda, A; Olthoff, Km

Lutz, Jt; Valentin-Gamazo, C; Gorlinger, K; Malago, M; Peters, J

Kang, Yg; Aggarwal, S; Virji, M; Pasculle, Aw; Lewis, Jh; Freeman, Ja; Martin, Lk

\begin{tabular}{|l|l|}
37 & 3,7 \\
\hline 37 & 3,7 \\
\hline 36 & 7,2 \\
\hline 36 & 3 \\
\hline 36 & 2,4 \\
\hline 36 & 2,25 \\
\hline 36 & 1,29 \\
\hline
\end{tabular}

\begin{tabular}{|c|c|c|c|c|c|}
\hline Deboer, Marieke T & 1 & 1.0 & Rayes, N & 1 & 1.0 \\
\hline Dejonge, J & 1 & 1.0 & Reich, DL & 1 & 1.0 \\
\hline Dellarocca, G & 1 & 1.0 & Roullet, S & 1 & 1.0 \\
\hline Dounıng, Lk & 1 & 1.0 & Sakai, T. & 1 & 1.0 \\
\hline Ellıs, Je & 1 & 1.0 & Schemmer, P. & 1 & 1.0 \\
\hline Faybik, P & 1 & 1.0 & Schroeder, RA & 1 & 1.0 \\
\hline Findlay, JY & 1 & 1.0 & Shangraw, Re & 1 & 1.0 \\
\hline Fitzsimons, MG & 1 & 1.0 & Shelly, Mp & 1 & 1.0 \\
\hline Ganter, Michael T. & 1 & 1.0 & Siniscalchi, A & 1 & 1.0 \\
\hline Glanemann, M & 1 & 1.0 & Sopher, M & 1 & 1.0 \\
\hline Goerlinger, K. & 1 & 1.0 & Starkel, P & 1 & 1.0 \\
\hline Haas, $T$. & 1 & 1.0 & Suriani, RJ & 1 & 1.0 \\
\hline Harding, SA & 1 & 1.0 & Swygert, Th & 1 & 1.0 \\
\hline Haruta, H & 1 & 1.0 & Takeı, Y & 1 & 1.0 \\
\hline Herbstreit, F. & 1 & 1.0 & Tamura, Sumihito & 1 & 1.0 \\
\hline Hilmi, I. A. & 1 & 1.0 & Taura, P & 1 & 1.0 \\
\hline Kaspar, M & 1 & 1.0 & Vanvugt, J. L. A. & 1 & 1.0 \\
\hline Kettner, SC & 1 & 1.0 & Watson, C. J. E. & 1 & 1.0 \\
\hline Khoury, Gf & 1 & 1.0 & Wax, David B & 1 & 1.0 \\
\hline Kim, Y. K & 1 & 1.0 & Weinrieb, RM & 1 & 1.0 \\
\hline Koelzow, H & 1 & 1.0 & Wikkelsoe, A. J & 1 & 1.0 \\
\hline Kuo, PC & 1 & 1.0 & Xia, Victor W & 1 & 1.0 \\
\hline Langle, F & 1 & 1.0 & & & \\
\hline
\end{tabular}


Table III. Distribution of first author affiliation for 103 studies with most citations related to liver transplantation in the anesthesia literature

\begin{tabular}{|c|c|c|}
\hline Affiliation & Frequency & $\%$ \\
\hline University of Pittsburgh Medical Center & 9 & 8.8 \\
\hline $\begin{array}{lll}\begin{array}{l}\text { Centre } \\
\text { Montreal }\end{array} & \text { Hospitalier } & \text { del'University } \\
& & \end{array}$ & 6 & 5.8 \\
\hline Baylor University medical center, Dallas & 6 & 5.8 \\
\hline University of California & 5 & 4.9 \\
\hline Universitats Klinik Essen, Germany & 3 & 2.8 \\
\hline University of Vienna & 3 & 2.8 \\
\hline Royal Free Hospital, London & 3 & 2.9 \\
\hline Addenbrookes Hospital & 3 & 2.8 \\
\hline Azienda Ospedaliera Universitaria Pisana & 2 & 1.9 \\
\hline $\begin{array}{lll}\begin{array}{l}\text { Centre } \\
\text { Bordeaux }\end{array} & \text { Hospitalo-Universitaire de } \\
\end{array}$ & 2 & 1.9 \\
\hline Erasmus University Medical Center & 2 & 1.0 \\
\hline Hospital Pellegrin CHU,Bordeaux & 2 & 1.9 \\
\hline Mayo Clinic & 2 & 1.9 \\
\hline Mount Sinai School of Medicine & 2 & 1.9 \\
\hline New England Medical Center, Boston & 2 & 1.9 \\
\hline Universite Paris-Sud, & 2 & 1.9 \\
\hline Paris Descartes University & 2 & 1.9 \\
\hline University of Heidelberg & 2 & 1.9 \\
\hline Albert Einstein Medical Center & 1 & 1.0 \\
\hline Austin Hospital, Melbourne & 1 & 1.0 \\
\hline $\begin{array}{l}\text { BethIsrael Deaconess Medical Center, } \\
\text { Boston }\end{array}$ & 1 & 1.0 \\
\hline Birmingham University & 1 & 1.0 \\
\hline Cairo University & 1 & 1.0 \\
\hline Charite Campus Virchow, Berlin, Germany & 1 & 1.0 \\
\hline Children's Hospital of Pittsburgh & 1 & 1.0 \\
\hline Cisanello Hospital & 1 & 1.0 \\
\hline Duke University School of Medicine & 1 & 1.0 \\
\hline Froedtert memorial lutheran hospital & 1 & 1.0 \\
\hline $\begin{array}{l}\text { HÃ'pitaux Universitaires, Strasbourg, } \\
\text { France }\end{array}$ & 1 & 1.0 \\
\hline Herlev Hospital, University of Copenhagen & 1 & 1.0 \\
\hline Hiroshima University School of Medicine & 1 & 1.0 \\
\hline Hospital Clinic, University of Barcelona & 1 & 1.0 \\
\hline
\end{tabular}

\begin{tabular}{|c|c|c|}
\hline Humboldt University Berlin & 1 & 1.0 \\
\hline Indiana University School of Medicine & 1 & 1.0 \\
\hline Jichi Medical School, Japan & 1 & 1.0 \\
\hline Lahey Clinic & 1 & 1.0 \\
\hline Massachusetts General Hospital & 1 & 1.0 \\
\hline Oregon Health\&Science University & 1 & 1.0 \\
\hline Princeps Dâ Espanya Hospital, Barcelona & 1 & 1.0 \\
\hline Ruprecht-Karls-University & 1 & 1.0 \\
\hline Rush Medical College & 1 & 1.0 \\
\hline St James's University Hospital & 1 & 1.0 \\
\hline St Vincent's Medical Center & 1 & 1.0 \\
\hline The Toronto Hospital & 1 & 1.0 \\
\hline $\begin{array}{l}\text { The University of Chicago Hospitals and } \\
\text { Clinics }\end{array}$ & 1 & 1.0 \\
\hline Toronto Western Hospital & 1 & 1.0 \\
\hline Triemli City Hospital Zurich & 1 & 1.0 \\
\hline Ucla school Of Medicine & 1 & 1.0 \\
\hline Univ of Groningen & 1 & 1.0 \\
\hline Universite $\hat{A}^{\prime}$ Catholique de Louvain & 1 & 1.0 \\
\hline University Children's Hospital Zurich & 1 & 1.0 \\
\hline University Drive C, Pittsburgh & 1 & 1.0 \\
\hline $\begin{array}{lll}\text { University } & \text { Hospital } & \text { Strasbourg- } \\
\text { Hautepierre } & & \end{array}$ & 1 & 1.0 \\
\hline $\begin{array}{l}\text { University of Colorado Health Sciences } \\
\text { Center }\end{array}$ & 1 & 1.0 \\
\hline University of Groningen, Netherlands & 1 & 1.0 \\
\hline University of Maryland, Baltimore & 1 & 1.0 \\
\hline University of Modena and Reggio Emilia & 1 & 1.0 \\
\hline University of North Carolina & 1 & 1.0 \\
\hline University of Pennsylvania & 1 & 1.0 \\
\hline University of Rochester & 1 & 1.0 \\
\hline University of Rome & 1 & 1.0 \\
\hline University of Tokyo & 1 & 1.0 \\
\hline University of Ulsan College of Medicine & 1 & 1.0 \\
\hline
\end{tabular}


Table IV. Distribution according to features of 103 studies with most citations related to liver transplantation in the anesthesia literature

\begin{tabular}{|c|c|c|c|c|c|c|}
\hline & Subgroup & $\mathrm{n}$ & $\begin{array}{c}\text { Total } \\
\text { Citiation } \\
\text { mean } \pm \text { SD }\end{array}$ & $p$ & $\begin{array}{c}\text { Annual } \\
\text { Citiation } \\
\text { mean } \pm \text { SD }\end{array}$ & $p$ \\
\hline \multirow{6}{*}{ Year } & $<1990$ & $\begin{array}{l}1 \\
1\end{array}$ & $\begin{array}{c}109,73 \pm 120 \\
20\end{array}$ & \multirow{6}{*}{$\begin{array}{c}0,697 \\
a\end{array}$} & $3,35 \pm 3,50$ & \multirow{6}{*}{$\begin{array}{c}<0,00 \\
1^{a}\end{array}$} \\
\hline & 1990-1994 & $\begin{array}{l}1 \\
5\end{array}$ & $82,93 \pm 92,88$ & & $3,13 \pm 3,40$ & \\
\hline & 1995-1999 & $\begin{array}{l}1 \\
7\end{array}$ & $59,94 \pm 20,23$ & & $2,70 \pm 0,94$ & \\
\hline & $2000-2004$ & $\begin{array}{l}2 \\
4\end{array}$ & $66,46 \pm 49,42$ & & $3,99 \pm 2,90$ & \\
\hline & 2005-2009 & $\begin{array}{l}2 \\
5\end{array}$ & $84,52 \pm 71,37$ & & $7,40 \pm 6,47$ & \\
\hline & $>2010$ & $\begin{array}{l}1 \\
1\end{array}$ & $57,91 \pm 16,81$ & & $9,35 \pm 3,32$ & \\
\hline \multirow{2}{*}{$\begin{array}{l}\text { Author's } \\
\text { Continen } \\
\text { ts }\end{array}$} & Europan & $\begin{array}{l}4 \\
4\end{array}$ & $85,07 \pm 80,19$ & \multirow{2}{*}{$\begin{array}{c}0,241 \\
\mathrm{~b}\end{array}$} & $6,49 \pm 5,96$ & \multirow{2}{*}{$0,009^{b}$} \\
\hline & Non-European & $\begin{array}{l}5 \\
9\end{array}$ & $69,02 \pm 58,02$ & & $3,86 \pm 2,79$ & \\
\hline \multirow{16}{*}{$\begin{array}{l}\text { Author's } \\
\text { Countries }\end{array}$} & USA & $\begin{array}{l}4 \\
7\end{array}$ & $69,23 \pm 63,46$ & & $3,46 \pm 2,75$ & \multirow{20}{*}{$0,018^{a}$} \\
\hline & France & $\begin{array}{l}1 \\
0\end{array}$ & $70,80 \pm 25,97$ & & $5,18 \pm 3,04$ & \\
\hline & England & 8 & $\begin{array}{c}111,0 \\
\pm 123,75\end{array}$ & & $6,11 \pm 5,26$ & \\
\hline & Germany & 7 & $80,43 \pm 88,17$ & & $6,07 \pm 5,61$ & \\
\hline & Canada & 6 & $82,33 \pm 33,31$ & & $7,09 \pm 2,25$ & \\
\hline & Italy & 5 & $63,40 \pm 30,02$ & & $5,62 \pm 3,77$ & \\
\hline & Netherlands & 5 & $79,20 \pm 51,95$ & & $9,02 \pm 5,55$ & \\
\hline & Japan & 3 & $68,00 \pm 20,66$ & 0,519 & $4,48 \pm 1,70$ & \\
\hline & Austria & 3 & $51,00 \pm 1,73$ & & $2,64 \pm 0,56$ & \\
\hline & Switzerland & 2 & $\begin{array}{c}206,0 \pm 240,4 \\
1\end{array}$ & & $\begin{array}{c}20,69 \pm 19,0 \\
7\end{array}$ & \\
\hline & Spain & 2 & $85,50 \pm 23,33$ & & $4,18 \pm 1,67$ & \\
\hline & Belgium & 1 & 82 & & 4,82 & \\
\hline & Australia & 1 & 46 & & 1,84 & \\
\hline & Denmark & 1 & 53 & & 6,63 & \\
\hline & Egypt & 1 & 37 & & 3,7 & \\
\hline & South Korea & 1 & 37 & & 3,7 & \\
\hline \multirow{4}{*}{$\begin{array}{l}\text { Article } \\
\text { type }\end{array}$} & $\begin{array}{l}\text { Prospective } \\
\text { Clinical }\end{array}$ & $\begin{array}{l}4 \\
6\end{array}$ & $74,48 \pm 65,81$ & \multirow{4}{*}{$\begin{array}{c}0,094 \\
a\end{array}$} & $4,55 \pm 3,43$ & \\
\hline & Retrospective & $\begin{array}{l}2 \\
2\end{array}$ & $62,82 \pm 23,52$ & & $5,17 \pm 3,50$ & \\
\hline & Case series & $\begin{array}{l}1 \\
2\end{array}$ & $50,92 \pm 15,29$ & & $2,40 \pm 1,33$ & \\
\hline & Rewiev & $\begin{array}{l}1 \\
1\end{array}$ & $\begin{array}{c}120,64 \pm 134 \\
97\end{array}$ & & $9,73 \pm 9,15$ & \\
\hline
\end{tabular}

\begin{tabular}{|c|c|c|c|c|c|c|}
\hline & RCT & 6 & $116,5 \pm 84,42$ & & $5,86 \pm 5,35$ & \\
\hline & Other & 3 & $44,0 \pm 10,53$ & & $2,68 \pm 0,83$ & \\
\hline & Experimental & 2 & $94,0 \pm 59,39$ & & $3,51 \pm 1,90$ & \\
\hline & Meta analysis & 1 & 50 & & 4,17 & \\
\hline \multirow{2}{*}{$\begin{array}{l}\text { Physician } \\
\text { Area }\end{array}$} & Anesthetist & $\begin{array}{l}8 \\
6\end{array}$ & $73,64 \pm 69,66$ & \multirow{2}{*}{$\begin{array}{c}0,320 \\
\mathrm{~b}\end{array}$} & $4,61 \pm 4,49$ & \multirow{2}{*}{$0,021^{b}$} \\
\hline & Non-Anesthetist & $\begin{array}{l}1 \\
7\end{array}$ & $87,18 \pm 62,87$ & & $6,89 \pm 4,81$ & \\
\hline \multirow{18}{*}{ Journal } & $\begin{array}{l}\text { Anesthesia And } \\
\text { Analgesia }\end{array}$ & $\begin{array}{l}3 \\
7\end{array}$ & $79,76 \pm 86,03$ & & $4,67 \pm 5,81$ & \\
\hline & $\begin{array}{l}\text { British Journal Of } \\
\text { Anaesthesia }\end{array}$ & $\begin{array}{l}1 \\
4\end{array}$ & $94,14 \pm 93,37$ & & $7,47 \pm 5,21$ & \\
\hline & $\begin{array}{l}\text { Liver } \\
\text { Transplantation }\end{array}$ & $\begin{array}{l}1 \\
0\end{array}$ & $63,30 \pm 32,78$ & & $4,65 \pm 2,92$ & \\
\hline & Anesthesiology & $\begin{array}{l}1 \\
0\end{array}$ & $74,20 \pm 38,17$ & & $2,69 \pm 1,34$ & \\
\hline & Transplantation & 7 & $\begin{array}{l}104,71 \\
\pm 82,82\end{array}$ & & $6,79 \pm 4,70$ & \\
\hline & $\begin{array}{l}\text { Journal Of } \\
\text { Cardiothoracic } \\
\text { And Vascular } \\
\text { Anesthesia }\end{array}$ & 6 & $69,67 \pm 38,49$ & & $4,61 \pm 3,33$ & \\
\hline & $\begin{array}{l}\text { Acta } \\
\text { Anaesthesiologica } \\
\text { Scandinavica }\end{array}$ & 3 & $47,00 \pm 8,71$ & & $4,38 \pm 1,99$ & \\
\hline & $\begin{array}{l}\text { European Journal } \\
\text { Of } \\
\text { Anaesthesiology }\end{array}$ & 2 & $45,0 \pm 5,65$ & & $2,90 \pm 0,03$ & \\
\hline & $\begin{array}{l}\text { Canadian Journal } \\
\text { Of Anaesthesia }\end{array}$ & 2 & $91,5 \pm 4,95$ & a & $5,78 \pm 0,71$ & $0,176^{a}$ \\
\hline & $\begin{array}{l}\text { Journal Of Clinical } \\
\text { Anesthesia }\end{array}$ & 2 & $53,5 \pm 2,12$ & & $2,57 \pm 0,44$ & \\
\hline & $\begin{array}{l}\text { Transplantation } \\
\text { Proceedings }\end{array}$ & 2 & $51,5 \pm 2,12$ & & $4,29 \pm 0,17$ & \\
\hline & Anaesthesia & 2 & $44,5 \pm 2,12$ & & $3,53 \pm 2,23$ & \\
\hline & $\begin{array}{l}\text { Pediatric } \\
\text { Transplantation }\end{array}$ & 1 & 82 & & 4,82 & \\
\hline & $\begin{array}{l}\text { Current Opinion in } \\
\text { Anesthesiology }\end{array}$ & 1 & 52 & & 4,73 & \\
\hline & $\begin{array}{l}\text { Transplant } \\
\text { İnternational }\end{array}$ & 1 & 49 & & 3,77 & \\
\hline & $\begin{array}{l}\text { American Journal } \\
\text { Of Transplantation }\end{array}$ & 1 & 42 & & 14 & \\
\hline & $\begin{array}{l}\text { Minerva } \\
\text { Anestesiologica }\end{array}$ & 1 & 36 & & 7,2 & \\
\hline & $\begin{array}{l}\text { Anaesthesia And } \\
\text { İntensive Care }\end{array}$ & 1 & 46 & & 1,84 & \\
\hline & USA & $\begin{array}{l}7 \\
7\end{array}$ & $76,14 \pm 67,86$ & & $4,65 \pm 4,68$ & \\
\hline & England & $\begin{array}{l}1 \\
8\end{array}$ & $83,17 \pm 84,35$ & & $6,52 \pm 4,93$ & \\
\hline Journal & & & & 0,249 & & $0380^{a}$ \\
\hline Countries & Swedish & 3 & $47,0 \pm 8,71$ & a & $4,38 \pm 1,99$ & 0,000 \\
\hline & Canada & 2 & $91,5 \pm 4,95$ & & $5,78 \pm 0,71$ & \\
\hline & Austria & 1 & 49 & & 3,77 & \\
\hline
\end{tabular}




\begin{tabular}{|c|c|c|c|c|c|c|}
\hline & Italy & 1 & 36 & & 7,2 & \\
\hline & Australia & 1 & 46 & & 1,84 & \\
\hline Continent & Europan & $\begin{array}{l}2 \\
3\end{array}$ & $74,91 \pm 75,94$ & & $6,15 \pm 4,47$ & \\
\hline $\begin{array}{l}\text { of the } \\
\text { journal }\end{array}$ & Non-European & $\begin{array}{l}8 \\
0\end{array}$ & $76,15 \pm 66,69$ & $\begin{array}{l}0,513 \\
-\quad b\end{array}$ & $4,65 \pm 4,60$ & $0,136^{\mathrm{b}}$ \\
\hline Journal & SCI & $\begin{array}{l}9 \\
5\end{array}$ & $77,96 \pm 70,83$ & 0,131 & $4,96 \pm 4,67$ & $0\ulcorner 10$ \\
\hline & SCI-E & 8 & $51,13 \pm 14,08$ & & $5,21 \pm 3,89$ & \\
\hline & Transfusion & $\begin{array}{l}1 \\
7\end{array}$ & $87,88 \pm 40,38$ & & $5,98 \pm 3,40$ & \\
\hline & $\begin{array}{l}\text { Thromboelastgrap } \\
\text { hy }\end{array}$ & $\begin{array}{l}1 \\
4\end{array}$ & $\begin{array}{c}139,79 \\
\pm 151,21\end{array}$ & & $9,07 \pm 8,48$ & \\
\hline & $\begin{array}{l}\text { Hemodynamic } \\
\text { Monitoring }\end{array}$ & $\begin{array}{l}1 \\
0\end{array}$ & $61,30 \pm 23,75$ & & $4,45 \pm 2,96$ & \\
\hline & $\begin{array}{l}\text { Anesthesia } \\
\text { Management }\end{array}$ & 8 & $55,25 \pm 25,09$ & & $2,72 \pm 1,11$ & \\
\hline & Surgery & 8 & $53,75 \pm 15,07$ & & $5,02 \pm 3,88$ & \\
\hline & Pharmacology & 8 & $56,0 \pm 13,33$ & & $2,27 \pm 0,69$ & \\
\hline & $\begin{array}{l}\text { Pulmonary } \\
\text { Hypertension }\end{array}$ & 8 & $57,63 \pm 13,26$ & & $2,72 \pm 0,79$ & \\
\hline & $\begin{array}{l}\text { Pulmonary } \\
\text { Thromboembolis }\end{array}$ & 6 & $43,83 \pm 6,61$ & & $2,90 \pm 1,83$ & \\
\hline Study & $\mathrm{m}$ & & & 0,097 & & $0,017 \mathrm{a}$ \\
\hline & Fluid Management & 4 & $6,25 \pm 26,92$ & & $3,89 \pm 1,35$ & \\
\hline & Perioperative care & 4 & $\begin{array}{c}106,75 \\
\pm 115,45\end{array}$ & & $6,48 \pm 6,64$ & \\
\hline & $\begin{array}{l}\text { Regional } \\
\text { Anesthesia, } \\
\text { Analgesia-Pain }\end{array}$ & 3 & $49,67 \pm 4,50$ & & $3,10 \pm 0,64$ & \\
\hline & Intensive Care & 3 & $71,0 \pm 34,64$ & & $5,08 \pm 4,34$ & \\
\hline & Renal Failure & 3 & $64,67 \pm 19,14$ & & $8,58 \pm 6,88$ & \\
\hline & History & 2 & $78,50 \pm 4,95$ & & $7,76 \pm 4,16$ & \\
\hline & $\begin{array}{l}\text { Hematology- } \\
\text { Oncology }\end{array}$ & 2 & $43,0 \pm 5,65$ & & $2,16 \pm 0,84$ & \\
\hline & Histopathology & 1 & 136 & & 4,86 & \\
\hline & Complication & 1 & 68 & & 2,13 & \\
\hline
\end{tabular}

a: Kruskal Wallis test, b: Mann-Whitney U test

\section{DISCUSSION}

The scientific research organization of the "Institute for Scientific Information (ISI)" was founded in America. The basic function of the Institute for Scientific Information is to determine journals to be included in the scope of the index and to periodically check these journals. Currently, ISI does not include all journals publishing scientific articles in the index. They select journals that abide by certain quality conditions. It also has a special scientific bibliographic search database for ISI, citations and index. It contains information about original articles published after 1945. Using the Web of Science, it is possible to reach valuable information like academic personnel citation statistics and mean citation indexes in the database $\mathrm{f}^{7,8}$. This information is requested for professional advancement and promotion.

The first bibliographic study by Garfield in 1987 was entitled "100 citation classics from the Journal of the American Medical Association" and published in the "Journal of the American Medical Association (JAMA)" 9 . Since then, many articles have investigated and presented the "most cited articles" not just in general journals but in specific journals ${ }^{10,11 .}$

Our study is the first to evaluate and analyze the top 103 articles with most citations related to liver transplantation in the anesthesia literature. The articles with most citations in the literature about this topic had 456 citations. The most cited studies related to the topic were about transfusion practice in liver transplantation. This was followed by articles about thromboelastography and hemodynamic monitoring. The study topic with highest total citations was thomboelastography with 139 citations.

There was no significant correlation determined between the continent of the journal and journal index with total and annual citation numbers for the articles. There was no statistically significant correlation found between the author's continent and total citation numbers. Additionally, authors from Europe had mean annual citation numbers that were significantly higher than authors from areas outside of Europe.

Ozbilgin et al. in a 2017 study of the top 100 articles with most citations about the topic of 
liver transplantation determined the mean citation number was 252.3. The most cited article was a study by Feng et al. entitled "Characteristics associated with liver graft failure: the concept of a donor risk index" with 677 citations. In the same study, of the total of 100 articles, 42 were prospective clinical studies, 25 were retrospective and ${ }^{13}$ were review studies ${ }^{12}$. McDowell et al. investigated articles with most citations in the field of pediatric liver transplantation and reported the mean citation number was $166^{13}$. Yllmaz et al. in a bibliometric study assessing the scientific performance of anesthesia clinics in Turkey determined the mean citation number was 9.9 and 376 of these studies were randomized controlled studies, 98 were observations and 66 were laboratory studies ${ }^{14}$. In our study the mean citation number was found to be 75.8. The most cited article was a study by Kang et al. with 456 citations. The studies included 46 prospective clinical studies, 22 retrospective and 12 case series. Of the 103 most cited articles, $92.2 \%$ were SCI index journals and $7.8 \%$ were SCI-E index journals. However, the mean annual citation number was higher for articles published in SCI-E journals. When the total citation numbers are examined, the most cited were review and randomized controlled studies (RCS), with least citations for case studies. This result is considered to be due to reviews and guidelines being at the top of the evidence pyramid while case studies are at the base. When the top-cited 103 articles are investigated, the country with most publications was the USA. The organizations with most articles were again on the American continent.

When study numbers are investigated according to year, 25 articles were published from 2005-2009, 24 studies from 2000-2004, with 11 studies after 2010 . When total citation numbers are investigated, the most citations were received by 11 studies published before
1990. Additionally, when the mean annual citation numbers are examined, as the years advanced, there was an increase observed in citation numbers. We think this result is affected by the developing information and technology in the field of liver transplantation. The study by Ozbilgin et al. observed an increase in mean annual numbers in spite of fewer publications in recent years ${ }^{12}$.

Research in Scandinavian countries in the field of anesthesiology and intensive care from 1981 to 2000,15 investigated the annual publication numbers in journals from Sweden, Norway, Finland and England over a 20-year period. Within this time, there was a significant increase in Finland and Norway compared to other countries and they reported that within these four countries, reference numbers increased in percentages within the last 20 years.

The first limitation of our study is that we used the WOS as search engine. It is known that different databases may display differences in citation numbers. Though additional citation numbers and mean annual citation numbers are traditional parameters to determine the scientific value of an article, the contribution of a study to science cannot be assessed using these parameters alone.

In conclusion, bibliographic articles created using scientific search engines illustrate the topics that researchers focus on. For healthy data analysis, it is recommended that these types of studies should be updated and rewritten at certain intervals.

Ethics Committee Approval: Hitit University Faculty of Medicine Clinical Research Ethics by the presidency of the board due to the design of the study board approval was not required.

Declaration of Conflicting Interests: The authors declare that they have no conflict of interest. 
Financial Disclosure: No financial support was received.

\section{REFERENCES}

1. Topal A,Celik JB. Anesthetic Management for Liver Transplantation. Turkiye Klinikleri J Anest Reanim-Special Topics. 2009; 2: 25-38

2. Raia S, Nery JR, Mies S. Liver transplantation from live donors. Lancet. 1989; 2: 497

3. Strong RW, Lynch SV, Ong TH, et al. Successful liver transplantation from a living donor to her son. N Engl J Med. 1990; 322: 1505-7

4. Hong SJ, Yoon DY, Cho YK, et al. Characteristic sand quality of radiologic randomized controlled trials: a bibliometric analysis between 1995 and 2014. Am J Roentgenol. 2016; 206: 917-923.

5. Ohba N, Nakao K, İsashiki Y, et al. The 100 most cited articles in ophthalmology journals. Arch Ophthalmol. 2007; 125: 952-60.

6. Thomson Scientific İsi Web of Knowledge: http://scientific.thomson.com/webofknowledg e.

7. Aminian A, Christopher R. Daigle, et al. Schauer, Citation classics: Top 50 cited articles in bariatric and metabolic surgery. Surgery for Obesity and Related Diseases. 2014; 10: 898905.

8. Bayramlar H, Cakici O, Karadag R, et al. The most frequently cited 100 Turkish articles in
Ophthalmic literature. Medeniyet Medical Journal. 2015; 30: 13-21.

9. Garfield E. 100 citation classics from the Journal of the American Medical Association. JAMA. 1987; 257: 52-9.

10. Paladugu R, Schein M, Gardezi S, et al. One hundred citation classics in general surgical journals. World J Surg. 2002; 26: 1099-105.

11. Lefaivre KA, Shadgan B, O’Brien PJ. 100 most cited articles in orthopaedic surgery. Clin Orthop Relat Res. 2011; 469: 1487-97.

12. M. Özbilgin, T. Ünek, T. Egeli, et al. The Most Frequently Cited 100 Articles in Liver Transplantation Literature. Transplantation Proceedings. 2017; 49: 551-61

13. McDowell DT, Darani A, Shun A, et al. A bibliometric analysis of pediatric liver transplantation publications. Pediatr Transplant. 2017; 21:e12913. https://doi.org/10.1111/petr.12913

14. Yılmaz HO, Babazade R, Turan OA, et al. Scientific publication performance of Turkish an aesthesia clinics in high impact factor international journals between 2005 and 2014: A bibliometric analysis. Turk J Anaesthesiol Reanim. 2017; 45: 16-25.

15. Skram U, Larsen $B$, İngwersen $P$, et al. Scandinavian research in anaesthesiology 1981-2000: visibility and impact in EU and world context. Acta Anaesthesiol Scand. 2004; 48: 1006-13. 